\title{
INHOMOGENEOUS PARABOLIC EQUATIONS ON UNBOUNDED METRIC MEASURE SPACES
}

\author{
KENNETH J. FALCONER, JIAXIN HU, AND YUHUA SUN
}

\begin{abstract}
Aвstract. We study inhomogeneous semilinear parabolic equations with source term $f$ independent of time $u_{t}=\Delta u+u^{p}+f(x)$ on a metric measure space, subject to the conditions that $f(x) \geq 0$ and $u(0, x)=\varphi(x) \geq 0$. By establishing Harnack-type inequalities in time $t$ and some powerful estimates, we give sufficient conditions for non-existence, local existence, and global existence of weak solutions. This paper generalizes previous results on Euclidean spaces to general metric measure spaces.
\end{abstract}

\section{Contents}

1. Introduction

2. Non-existence of solutions

3. Existence of solutions

4. Regularity

References

\section{INTRODUCTION}

In recent years, the study of PDEs on self-similar fractals has attracted increasing interest, see for example [7, 8, 9, 13, 14]. In this paper we investigate a class of nonlinear diffusions with source terms on general metric measure spaces. Diffusion is of fundamental importance in many areas of physics, chemistry, and biology. Applications of diffusion include: (1) Sintering, i.e. making solid materials from powder (powder metallurgy, production of ceramics); (2) Catalyst design in the chemical industry; (3) Steel can be diffused (e.g. with carbon or nitrogen) to modify its properties; (4) Doping during production of semiconductors; (5) The well-known BlackScholes Model in Financial Mathematics that is closely related to option pricing can be transformed to a parabolic equation.

1991 Mathematics Subject Classification. Primary: 35K05, Secondary: 28A80, 60J35.

Key words and phrases. diffusion equation; heat kernel; metric measure space.

JH was supported by NSFC (Grant No. 11071138).

KJF thanks the Department of Mathematical Sciences, Tsinghua University, for their hospitality. 
Let $(M, d, \mu)$ be a metric measure space, that is, $(M, d)$ is a locally compact separable metric space and $\mu$ is a Radon measure on $M$ with full support. We consider the following nonlinear diffusion equation with a source term $f$ on $(M, d, \mu)$ :

$$
u_{t}=\Delta u+u^{p}+f(x), t>0 \text { and } x \in M,
$$

with initial value

$$
u(0, x)=\varphi(x),
$$

where $p>1$ and $f, \varphi: M \rightarrow \mathbb{R}$ are non-negative measurable functions. With an appropriate interpretation of weak solutions of (1.1) on $(M, d, \mu)$, we shall investigate the non-existence (or blow-up) of solutions, the local and global existence of weak solutions to (1.1)-(1.2), as well as the regularity of these solutions. Although we were partially motivated by a series of earlier papers [1, 9, 10, 11, 15, 16, 17], there are new ideas in this paper. In particular, we have used the theory of heat kernels on metric measure spaces.

Recall the definition of the heat kernel which will be central to our approach. A function $k(\cdot, \cdot, \cdot): \mathbb{R}_{+} \times M \times M \rightarrow \mathbb{R}$ is called a heat kernel if the following conditions $(k 1)-(k 4)$ are fulfilled: for $\mu$-almost all $(x, y) \in M \times M$ and for all $t, s>0$,

(k1) Markov property: $k(t, x, y)>0$, and $\int_{M} k(t, x, y) d \mu(y) \leq 1$;

( $k 2)$ symmetry: $k(t, x, y)=k(t, y, x)$;

(k3) semigroup property: $k(s+t, x, z)=\int_{M} k(s, x, y) k(t, y, z) d \mu(y)$;

$(k 4)$ normalization: for all $f \in L^{2}(M, \mu)$

$$
\lim _{t \rightarrow 0^{+}} \int_{M} k(t, x, y) f(y) d \mu(y)=f(x) \text { in the } L^{2}(M, \mu) \text {-norm. }
$$

We assume that the heat kernel $k(t, x, y)$ considered in this paper is jointly continuous in $x, y$, and hence the above formulae in $(k 1)-(k 4)$ hold for every $(x, y) \in M \times M$.

Two typical examples of heat kernels in $\mathbb{R}^{n}$ are the Gauss-Weierstrass and the Cauchy-Poisson kernels:

$$
\begin{aligned}
& k(t, x, y)=\frac{1}{(4 \pi t)^{n / 2}} \exp \left(-\frac{|x-y|^{2}}{4 t}\right) \\
& k(t, x, y)=\frac{C_{n}}{t^{n}}\left(1+\frac{|x-y|^{2}}{t^{2}}\right)^{-(n+1) / 2} \quad\left(C_{n}=\frac{\Gamma\left(\frac{1}{2}(n+1)\right)}{\pi^{(n+1) / 2}}\right) .
\end{aligned}
$$

Jointly continuous sub-Gaussian heat kernels exist on many basic fractals, for example, on the Sierpínski gasket, see Barlow and Perkins [5], and on Sierpínski carpets, see Barlow and Bass [3, 2]. For other fractals see [12, 13]. For non-sub-Gaussian heat kernels, see [4, 6].

A heat kernel $k$ is called conservative if it satisfies

(k5) conservative property: $\int_{M} k(t, x, y) d \mu(y)=1$, for all $t>0$ and all $x \in M$.

We will also assume that the heat kernel satisfies the following estimates 
(k6) two-sided bounds: there exist constants $\alpha, \beta>0$ such that for all $t>0$ and all $x, y \in M$,

$$
\frac{1}{t^{\alpha / \beta}} \Phi_{1}\left(\frac{d(x, y)}{t^{1 / \beta}}\right) \leq k(t, x, y) \leq \frac{1}{t^{\alpha / \beta}} \Phi_{2}\left(\frac{d(x, y)}{t^{1 / \beta}}\right)
$$

where $\Phi_{1}$ and $\Phi_{2}$ are strictly positive and non-increasing functions on $[0, \infty)$.

It turns out that the parameter $\alpha$ in (1.3) is the fractal dimension, and $\beta$ is the walk dimenison of $M$, see [11].

Two-sided estimates (1.3) hold on various fractals where

$$
\Phi_{i}(s)=C_{i} \exp \left(-c_{i} s^{\beta /(\beta-1)}\right) \text { (for all } s \geq 0 \text { ) }
$$

for constants $C_{i}, c_{i}>0(i=1,2)$ and $\beta>2$ is the walk dimension.

To prove the regularity of solutions, we need to assume that the heat kernel $k$ is Hölder continuous in the space variables:

(k7) Hölder continuity: there exist constants $L>0, v \geq 1$ and $0<\sigma \leq 1$ such that

$$
\left|k\left(t, x_{1}, y\right)-k\left(t, x_{2}, y\right)\right| \leq L t^{-v} d\left(x_{1}, x_{2}\right)^{\sigma}
$$

for all $t>0$ and all $x_{1}, x_{2}, y \in M$.

Given a heat kernel $k$, the operator $\Delta$ in (1.1) is interpreted as the infinitesimal generator of the heat semigroup $\left\{K_{t}\right\}_{t \geq 0}$ in $L^{2}:=L^{2}(M, \mu)$. Thus we let

$$
K_{t} g(x)=\int_{M} k(t, x, y) g(y) d \mu(y)\left(t>0, g \in L^{2}\right),
$$

and define $\Delta$ by

$$
\Delta g=\lim _{t \downarrow 0} \frac{K_{t} g-g}{t} \text { (in } L^{2} \text {-norm). }
$$

Observe that $\left\{K_{t}\right\}_{t>0}$ is a strongly continuous and contractive semigroup in $L^{2}$, that is, for all $s, t \geq 0$ and all $g \in L^{2}$,

$$
\begin{aligned}
K_{s+t} & =K_{s} K_{t}, \\
\lim _{t \rightarrow 0^{+}}\left\|K_{t} g-g\right\|_{2} & =0, \\
\left\|K_{t} \phi\right\|_{q} & \leq\|\phi\|_{q}(\text { for all } 1 \leq q \leq \infty) .
\end{aligned}
$$

The domain of the operator $\Delta$ is dense in $L^{2}$.

A function $u(t, x)$ is termed a weak solution to (1.1)-(1.2) if it satisfies the following integral equation

$$
u(t, x)=K_{t} \varphi(x)+\int_{0}^{t} K_{\tau} f(x) d \tau+\int_{0}^{t} K_{t-\tau} u^{p}(\tau, x) d \tau,
$$

where $K_{t}$ is the heat semigroup defined in (1.4).

The structure of this paper is as follows. In Section 2 , we show the nonexistence of weak solution to (1.1)-(1.2). In Section 3, we obtain sufficient conditions for the local and global existence of solutions for a range of parameters $p$, source terms $f$ and intial values $\varphi$. The critical exponents $p$ 
depend only on the fractal dimension $\alpha$ and the walk dimension $\beta$. Finally, in Section 4, we investigate the Hölder continuity of weak solutions.

Notation. The letters $C, C_{i}(i=1,2, \ldots)$ denote positive constants whose values are unimportant and may differ at different occurrences.

\section{NON-EXISTENCE OF SOLUTIONS}

In this section we give sufficient conditions for the non-existence of essentially bounded solutions. The exponents $p=1+\beta / \alpha$ (where $\alpha, \beta>0$ ), and $p=\alpha /(\alpha-\beta)$, where $\alpha>\beta>0$ occur in the heat kernel bounds (1.3), play a crucial rôle in our analysis, see Theorem 2.2 . First, we establish Lemma 2.1, where condition $(k 6)$ is our only assumption on the heat kernel $k$ (we do not need the conservative property of $k$ at this stage.)

The following properties the functions $\Phi_{1}$ and $\Phi_{2}$ in condition ( $k 6$ ) may or may not hold: there exist positive constants $a_{i}, b_{i}$ and $c_{i}$ such that, for all $s, t \geq 0$,

$$
\begin{aligned}
\Phi_{1}(s) & \geq a_{1} \Phi_{2}\left(a_{2} s\right), \\
\Phi_{2}(s+t) & \geq b_{1} \Phi_{2}\left(b_{2} s\right) \Phi_{2}\left(b_{3} t\right), \\
\Phi_{1}^{p}(s) & \geq c_{1} \Phi_{2}\left(c_{2} s\right) .
\end{aligned}
$$

Note that if (2.1) holds, then $0<a_{1} \leq 1$ by letting $s=0$ and using the fact that $\Phi_{2}(0) \geq \Phi_{1}(0)$. Without loss of generality, we may assume that $a_{2}>1$ in (2.1), since if (2.1) holds for some $a_{2} \leq 1$, it also holds for any constant $a_{2}>1$ by the monotonicity of $\Phi_{2}$.

The Gauss-type functions $\Phi_{1}$ and $\Phi_{2}$

$$
\begin{aligned}
& \Phi_{1}(s)=C_{1} \exp \left(-C_{2} s^{\gamma}\right), \\
& \Phi_{2}(s)=C_{3} \exp \left(-C_{4} s^{\gamma}\right), s \geq 0,
\end{aligned}
$$

for constants $\gamma>0$ and $C_{i}>0(1 \leq i \leq 4)$ satisfy properties (2.1)-(2.3). The Cauchy-type functions

$$
\begin{aligned}
& \Phi_{1}(s)=C_{1}(1+s)^{-\gamma}, \\
& \Phi_{2}(s)=C_{2}(1+s)^{-\gamma}, s \geq 0
\end{aligned}
$$

for constants $\gamma>0$ and $C_{i}>0(i=1,2)$, satisfy properties (2.1) and (2.2), but not (2.3) if $p>1$.

Condition $(k 6)$ and inequality (2.1) lead to the following key lemma.

Lemma 2.1. Assume that the heat kernel $k$ satisfies condition ( $k 6)$ and (2.1). Then, for all non-negative measurable functions $g$ on $M$ and for all $t>$ $0, x \in M$,

$$
\begin{aligned}
K_{t} g(x) & \geq A_{1} K_{B t} g(x), \\
\int_{0}^{t} K_{\tau} g(x) d \tau & \geq A_{2} t K_{B^{2} t} g(x),
\end{aligned}
$$


where $A_{1}=a_{1} a_{2}^{-\alpha}<1, A_{2}=a_{1} a_{2}^{-2 \alpha}\left(1-a_{2}^{-\beta}\right)<1$ and $B=a_{2}^{-\beta}<1$. Consequently, for all non-negative measurable functions $\varphi$,

$$
K_{t} \varphi(x)+\int_{0}^{t} K_{\tau} g(x) d \tau \geq A\left[K_{B_{1}} \varphi(x)+t K_{B_{1}} t g(x)\right],
$$

where $A=\min \left\{A_{1}^{2}, A_{2}\right\}<1$ and $B_{1}=B^{2}=a_{2}^{-2 \beta}$.

Proof. It follows from condition $(k 6)$ and (2.1) that

$$
\begin{aligned}
K_{t} g(x) & =\int_{M} k(t, x, y) g(y) d \mu(y) \\
& \geq \int_{M} \frac{1}{t^{\alpha / \beta}} \Phi_{1}\left(\frac{d(x, y)}{t^{1 / \beta}}\right) g(y) d \mu(y) \\
& \geq a_{1} \int_{M} \frac{1}{t^{\alpha / \beta}} \Phi_{2}\left(a_{2} \frac{d(x, y)}{t^{1 / \beta}}\right) g(y) d \mu(y) .
\end{aligned}
$$

which gives that, using $(k 6)$ again,

$$
\begin{aligned}
K_{t} g(x) & \geq a_{1} a_{2}^{-\alpha} \int_{M} k\left(a_{2}^{-\beta} t, x, y\right) g(y) d \mu(y) \\
& =a_{1} a_{2}^{-\alpha} K_{a_{2}^{-\beta} t} g(x)=A_{1} K_{B t} g(x),
\end{aligned}
$$

proving (2.6).

To show (2.7), we see from (2.9) that for all $\tau \in\left[a_{2}^{-\beta} t, t\right]$, using the monotonicity of $\Phi_{2}$ and condition $(k 6)$,

$$
\begin{aligned}
K_{\tau} g(x) & \geq a_{1} \int_{M} \frac{1}{\tau^{\alpha / \beta}} \Phi_{2}\left(a_{2} \frac{d(x, y)}{\tau^{1 / \beta}}\right) g(y) d \mu(y) \\
& \geq a_{1} \int_{M} \frac{1}{t^{\alpha / \beta}} \Phi_{2}\left(a_{2} \frac{d(x, y)}{\left(a_{2}^{-\beta} t\right)^{1 / \beta}}\right) g(y) d \mu(y) \\
& \geq a_{1} a_{2}^{-2 \alpha} \int_{M} k\left(a_{2}^{-2 \beta} t, x, y\right) g(y) d \mu(y) \\
& =a_{1} a_{2}^{-2 \alpha} K_{B^{2} t} g(x) .
\end{aligned}
$$

Therefore,

$$
\begin{aligned}
\int_{0}^{t} K_{\tau} g(x) d \tau & \geq \int_{a_{2}^{-\beta} t}^{t} K_{\tau} g(x) d \tau \\
& \geq \int_{a_{2}^{-\beta} t}^{t} a_{1} a_{2}^{-2 \alpha} K_{B^{2} t} g(x) d \tau \\
& =a_{1} a_{2}^{-2 \alpha}\left(1-a_{2}^{-\beta}\right) t K_{B^{2} t} g(x),
\end{aligned}
$$

proving (2.7).

Finally, replacing $t$ by $B t$, we see from (2.6) that $K_{B t} \varphi(x) \geq A_{1} K_{B^{2} t} \varphi(x)=$ $A_{1} K_{B_{1} t} \varphi(x)$, and thus

$$
K_{t} \varphi(x) \geq A_{1} K_{B t} \varphi(x) \geq A K_{B_{1} t} \varphi(x) .
$$


Adding (2.7) and (2.10), we obtain (2.8).

Lemma 2.1 gives the following estimate (2.11) that plays an important rôle in proving the non-existence of global bounded solutions.

Theorem 2.2. Assume that the heat kernel $k$ satisfies conditions $(k 6)$ and (2.1). Let $u(t, x)$ be a non-negative essentially bounded solution of (1.7) in $(0, T) \times M$. Then, for all $(t, x) \in(0, T) \times M$,

$$
t^{1 /(p-1)} K_{B_{1} t} \varphi(x)+t^{p /(p-1)} K_{B_{1} t} f(x) \leq C_{1},
$$

where $B_{1}=a_{2}^{-2 \beta}$ as before, and $C_{1}$ depends only on $p$ (and in particular is independent of $T, \varphi$ and $f$ ).

Proof. Observe that by condition $(k 1)$ and using a weighted Hölder inequality, for all $t>0, x \in M$ and for all non-negative functions $g$,

$$
\begin{aligned}
K_{t}\left(g^{p}\right)(x) & =\int_{M} k(t, x, y) g^{p}(y) d \mu(y) \\
& \geq\left[\int_{M} k(t, x, y) g(y) d \mu(y)\right]^{p}=\left[K_{t} g(x)\right]^{p} .
\end{aligned}
$$

It follows from (1.7) and (2.10) that

$$
\begin{aligned}
u(t, x) & \geq \int_{0}^{t} K_{t-\tau} u^{p}(\tau, x) d \tau \\
& \geq A \int_{0}^{t} K_{B_{1}(t-\tau)} u^{p}(\tau, x) d \tau \\
& \geq A \int_{0}^{t}\left[K_{B_{1}(t-\tau)} u(\tau, x)\right]^{p} d \tau .
\end{aligned}
$$

From (1.7) and (2.8), we see that

$$
\begin{aligned}
u(t, x) & \geq K_{t} \varphi(x)+\int_{0}^{t} K_{\tau} f(x) d \tau \\
& \geq A\left(K_{B_{1} t} \varphi(x)+t K_{B_{1}} f(x)\right)
\end{aligned}
$$

Starting from (2.13), we shall apply (2.12) repeatedly to deduce the desired inequality (2.11). Indeed, we obtain from (2.12) and (2.13) that, using the semigroup property (1.6) of $\left\{K_{t}\right\}_{t \geq 0}$ and the elementary inequality $(a+b)^{p} \geq$ 
$a^{p}+b^{p}$ for all $p \geq 1$ and $a, b \geq 0$,

$$
\begin{aligned}
u(t, x) & \geq A \int_{0}^{t}\left[K_{B_{1}(t-\tau)} u(\tau, x)\right]^{p} d \tau \\
& \geq A \int_{0}^{t}\left[K_{B_{1}(t-\tau)}\left\{A\left(K_{B_{1} \tau} \varphi+\tau K_{B_{1} \tau} f\right)\right\}(x)\right]^{p} d \tau \\
& =A^{p+1} \int_{0}^{t}\left[K_{B_{1} t} \varphi(x)+\tau K_{B_{1} t} f(x)\right]^{p} d \tau \\
& \geq A^{p+1}\left\{t\left(K_{B_{1} t} \varphi(x)\right)^{p}+\int_{0}^{t} \tau^{p}\left(K_{B_{1} t} f(x)\right)^{p} d \tau\right\} \\
& =A^{p+1}\left\{t\left(K_{B_{1} t} \varphi(x)\right)^{p}+\frac{1}{1+p} t^{1+p}\left(K_{B_{1} t} f(x)\right)^{p}\right\} .
\end{aligned}
$$

Repeating the above procedure, we obtain that for all $n \geq 1$,

$$
\begin{aligned}
u(t, x) \geq & A^{1+p+\cdots+p^{n}}\left\{\frac{t^{1+p+\cdots+p^{n-1}}\left[K_{B t} \varphi(x)\right]^{p^{n}}}{(1+p)^{p^{n-2}}\left(1+p+p^{2}\right)^{p^{n-3}} \cdots\left(1+p+\cdots+p^{n-1}\right)}\right. \\
& \left.+\frac{t^{1+p+\cdots+p^{n}}\left[K_{B t} f(x)\right]^{p^{n}}}{(1+p)^{p^{n-1}}\left(1+p+p^{2}\right)^{p^{n-2}} \cdots\left(1+p+\cdots+p^{n}\right)}\right\} .
\end{aligned}
$$

It follows that

$$
\begin{aligned}
& A^{\left(p^{n+1}-1\right) /(p-1) p^{n}} t^{\left(p^{n}-1\right) /(p-1) p^{n}} K_{B t} \varphi(x) \\
& \leq u(t, x)^{p^{-n}} \prod_{i=2}^{n}\left(1+p+\cdots+p^{i-1}\right)^{p^{-i}}, \\
& A^{\left(p^{n+1}-1\right) /(p-1) p^{n}} t^{\left(p^{n+1}-1\right) /(p-1) p^{n}} K_{B t} f(x) \\
& \leq u(t, x)^{p^{-n}} \prod_{i=1}^{n}\left(1+p+\cdots+p^{i}\right)^{p^{-i}} .
\end{aligned}
$$

Since

$$
\begin{aligned}
\log \prod_{i=2}^{n}\left(1+p+\cdots+p^{i-1}\right)^{p^{-i}} & \leq \sum_{i=2}^{\infty} \frac{1}{p^{i}} \log \left(i p^{i}\right)<+\infty, \\
\log \prod_{i=1}^{n}\left(1+p+\cdots+p^{i}\right)^{p^{-i}} & \leq \sum_{i=1}^{\infty} \frac{1}{p^{i}} \log \left((i+1) p^{i}\right)<+\infty,
\end{aligned}
$$

and that $u(t, x)$ is essentially bounded on $(0, T) \times M$, we pass to the limit as $n \rightarrow \infty$ in (2.14) and (2.15), and conclude that

$$
\begin{aligned}
& t^{1 /(p-1)} K_{B t} \varphi(x) \leq C_{1} / 2 \\
& t^{p /(p-1)} K_{B t} f(x) \leq C_{1} / 2
\end{aligned}
$$

for some $C_{1}>0$. Adding (2.16) and (2.17), we obtain (2.11).

We are now in a position to obtain the main results of this section. 
Theorem 2.3. Assume that the heat kernel $k$ satisfies conditions $(k 6)$ and (2.1). Then the problem (1.1)-(1.2) does not have any essentially bounded global solution in each of the following cases:

(i) if $p<1+\frac{\beta}{\alpha}$ and if either $\varphi(x) \geqq 0$ or $f(x) \geqq 0$.

(ii) if $\alpha \leq \beta$ and if $f(x) \geqq 0$;

(iii) if $\alpha>\beta$ and $p<\frac{\alpha}{\alpha-\beta}\left(>1+\frac{\beta}{\alpha}\right)$ and if $f(x) \geqq 0$.

Proof. We prove the results by contradiction. Assume that $u(t, x)$ is a non-negative essentially bounded global solution. Replacing $B_{1} t$ by $t$, we see from (2.11) that for all $x \in M$ and $t>0$,

$$
t^{1 /(p-1)} K_{t} \varphi(x)+t^{p /(p-1)} K_{t} f(x) \leq C_{1},
$$

where $0<C_{1}<\infty$ is independent of $\varphi$ and $f$.

Proof of Case (i): If $\varphi(x) \geqq 0$, we see from ( $k 6)$, using Fatou’s lemma, that

$$
\begin{aligned}
\liminf _{t \rightarrow \infty} t^{\alpha / \beta} K_{t} \varphi(x) & \geq \liminf _{t \rightarrow \infty} \int_{M} \Phi_{1}\left(\frac{d(x, y)}{t^{1 / \beta}}\right) \varphi(y) d \mu(y) \\
& \geq C_{2} .
\end{aligned}
$$

where $C_{2}=1$ if $\|\varphi\|_{1}=\infty$, and $C_{2}=\Phi_{1}(0)\|\varphi\|_{1}$ if $\|\varphi\|_{1}<\infty$. However, as $1 /(p-1)>\alpha / \beta$, this is impossible by using (2.18). Hence, (1.1)-(1.2) does not have any global essentially bounded solution.

If $f(x) \geqq 0$, observe that $u\left(t+t_{0}, x\right)$ is a weak solution of (1.7) with initial data $\varphi(x)=u\left(t_{0}, x\right)$. We may find $t_{0}>0$ such that $u\left(t_{0}, x\right) \geqq 0$. Repeating the above argument, we again see that (1.1)-(1.2) does not have any global essentially bounded solution.

Proof of Case (ii): Observe that by (1.7) and (2.7),

$$
u(t, x) \geq \int_{0}^{t} K_{\tau} f(x) d \tau \geq A_{2} t K_{B_{1} t} f(x) .
$$

We distinguish two cases: $\alpha<\beta$ and $\alpha=\beta$.

- The case $\alpha<\beta$. It follows from (2.19) and (k6) that

$$
\begin{aligned}
\liminf _{t \rightarrow \infty} t^{(\alpha / \beta)-1} u(t, x) & \geq A_{2} \liminf _{t \rightarrow \infty} t^{\alpha / \beta} K_{B_{1} t} f(x) \\
& \geq A_{2} \liminf _{t \rightarrow \infty} t^{\alpha / \beta} \int_{M} \frac{1}{\left(B_{1} t\right)^{\alpha / \beta}} \Phi_{1}\left(\frac{d(x, y)}{\left(B_{1} t\right)^{1 / \beta}}\right) f(y) d \mu(y) \\
& \geq C_{3},
\end{aligned}
$$

where $C_{3}=1$ if $\|f\|_{1}=\infty$ and $C_{3}=A_{2} B_{1}^{-\alpha / \beta} \Phi_{1}(0)>0$ if $\|f\|_{1}<\infty$. However, since $u$ is globally essentially bounded and $\alpha / \beta<1$, we see

$$
\liminf _{t \rightarrow \infty} t^{(\alpha / \beta)-1} u(t, x)=0,
$$

a contradiction. 
- The case $\alpha=\beta$. For $t>1$, it follows from (k6), (2.1) and the monotonicity of $\Phi_{2}$ that

$$
\begin{aligned}
u(t, x) & \geq \int_{0}^{t} K_{\tau} f(x) d \tau \\
& \geq \int_{0}^{t} d \tau \int_{M} \tau^{-1} \Phi_{1}\left(\frac{d(x, y)}{\tau^{1 / \beta}}\right) f(y) d \mu(y) \\
& \geq a_{1} \int_{1}^{t} d \tau \int_{M} \tau^{-1} \Phi_{2}\left(a_{2} \frac{d(x, y)}{\tau^{1 / \beta}}\right) f(y) d \mu(y) \\
& \geq a_{1} \int_{1}^{t} \tau^{-1} d \tau \int_{M} \Phi_{2}\left(a_{2} d(x, y)\right) f(y) d \mu(y) .
\end{aligned}
$$

Since $f(x) \geqq 0$, we can find a point $x \in M$ such that

$$
\int_{M} \Phi_{2}\left(a_{2} d(x, y)\right) f(y) d \mu(y)>0 .
$$

Passing to the limit as $t \rightarrow \infty$ in (2.21) this contradicts that $u$ is globally essentially bounded.

Proof of Case (iii): It follows from (2.18) and $(k 6)$ that

$$
\begin{aligned}
\liminf _{t \rightarrow \infty} C_{1} t^{\alpha / \beta-p /(p-1)} & \geq \liminf _{t \rightarrow \infty} t^{\alpha / \beta} K_{t} f(x) \\
& \geq \liminf _{t \rightarrow \infty} \int_{M} \Phi_{1}\left(\frac{d(x, y)}{t^{1 / \beta}}\right) f(y) d \mu(y) \\
& \geq C_{4},
\end{aligned}
$$

where $C_{4}=1$ if $\|f\|_{1}=\infty$ and $C_{4}=\Phi_{1}(0)\|f\|_{1}$ if $\|f\|_{1}<\infty$. However, this is impossible since $\frac{\alpha}{\beta}-\frac{p}{p-1}<0$. The proof is complete.

In Theorem 2.3, we do not know in general if there exists any essentially bounded global solution for two the critical cases $p=1+\frac{\beta}{\alpha}(\alpha, \beta>0)$ and $p=\frac{\alpha}{\alpha-\beta}(\alpha>\beta>0)$.

However, Theorem 2.3 (i) may be improved to include the critical exponent $p=1+\frac{\beta}{\alpha}$ under further assumptions (2.2) and (2.3) on the heat kernel $k$. We first need the following property.

Proposition 2.4. If $\Phi_{2}$ satisfies (2.2), then for all $t>0$ and all $x, y \in M$,

$$
\frac{\Phi_{2}\left(d(x, y) t^{-1 / \beta}\right)}{\Phi_{2}\left(b_{2} d(x, 0) t^{-1 / \beta}\right)} \geq b_{1} \Phi_{2}\left(b_{3} d(y, 0) t^{-1 / \beta}\right),
$$

where the constants $b_{i}(i=1,2,3)$ are as in (2.2).

Proof. Since $\Phi_{2}$ is strictly positive and decreasing on $[0, \infty)$ and $d(x, y) \leq$ $d(x, 0)+d(y, 0)$, we have

$$
\Phi_{2}\left(d(x, y) t^{-1 / \beta}\right) \geq \Phi_{2}\left(d(x, 0) t^{-1 / \beta}+d(y, 0) t^{-1 / \beta}\right) .
$$

It follows from (2.2) that

$$
\Phi_{2}\left(d(x, 0) t^{-1 / \beta}+d(y, 0) t^{-1 / \beta}\right) \geq b_{1} \Phi_{2}\left(b_{2} d(x, 0) t^{-1 / \beta}\right) \Phi_{2}\left(b_{3} d(y, 0) t^{-1 / \beta}\right),
$$


which combines with (2.23) to give (2.22).

Theorem 2.5. Assume that the heat kernel $k$ satisfies conditions $(k 5),(k 6)$ and (2.1), (2.2) and (2.3). Then (1.1)-(1.2) does not have any essentially bounded global solutions if $p \leq 1+\frac{\beta}{\alpha}$ and if either $\varphi(x) \geqq 0$ or $f(x) \geqq 0$.

Proof. In view of Theorem 2.3 (i) it is enough to consider the critical exponent $p=1+\beta / \alpha$. We only consider the case $\varphi(x) \geqq 0$ (the case $f(x) \geqq 0$ may be treated in a similar way). Then (2.18) becomes

$$
t^{\alpha / \beta} K_{t} \varphi(x)+t^{1+\alpha / \beta} K_{t} f(x) \leq C_{1} .
$$

From condition $(k 6)$

$$
\int_{M} \varphi(y) d \mu(y) \leq C_{2}
$$

where $C_{2}=C_{1} / \Phi_{1}(0)$. For any $t_{0}>0$, the function $v(t, x) \equiv u\left(t+t_{0}, x\right)$ is a weak solution to (1.7) with initial data $\varphi(x)=u\left(t_{0}, x\right)$. Repeating the procedure of (2.24), we have that for all $t_{0}>0$,

$$
\int_{M} u\left(t_{0}, y\right) d \mu(y) \leq C_{2}
$$

We claim that there exist positive constants $\gamma, \rho$ possibly depending on $t_{0}$ and $\varphi$ such that, for all $x \in M$,

$$
u\left(t_{0}, x\right) \geq \rho k(\gamma, x, 0) .
$$

To see this, observe that

$$
\Phi_{2}\left(d(x, 0) \gamma^{-1 / \beta}\right) \geq k(\gamma, x, 0) \gamma^{\alpha / \beta},
$$

and thus, using (2.22) and setting $\gamma=\left(a_{1} b_{2}\right)^{-\beta} t_{0}$,

$$
\begin{aligned}
\Phi_{2}\left(a_{2} d(x, y) t_{0}^{-1 / \beta}\right) & \geq b_{1} \Phi_{2}\left(a_{1} b_{3} d(y, 0) t_{0}^{-1 / \beta}\right) \Phi_{2}\left(a_{1} b_{2} d(x, 0) t_{0}^{-1 / \beta}\right) \\
& \geq b_{1} \Phi_{2}\left(a_{1} b_{3} d(y, 0) t_{0}^{-1 / \beta}\right) k(\gamma, x, 0) \gamma^{\alpha / \beta} .
\end{aligned}
$$

Using (1.7) and (2.1),

$$
\begin{aligned}
u\left(t_{0}, x\right) & \geq \int_{M} k\left(t_{0}, x, y\right) \varphi(y) d \mu(y) \\
& \geq t_{0}^{-\alpha / \beta} \int_{M} \Phi_{1}\left(d(x, y) t_{0}^{-1 / \beta}\right) \varphi(y) d \mu(y) \\
& \geq a_{1} t_{0}^{-\alpha / \beta} \int_{M} \Phi_{2}\left(a_{2} d(x, y) t_{0}^{-1 / \beta}\right) \varphi(y) d \mu(y) \\
& \geq a_{1} b_{1}\left(\frac{\gamma}{t_{0}}\right)^{\alpha / \beta} k(\gamma, x, 0) \int_{M} \Phi_{2}\left(a_{1} b_{3} d(y, 0) t_{0}^{-1 / \beta}\right) \varphi(y) d \mu(y),
\end{aligned}
$$

and hence, inequality (2.26) holds by setting

$$
\rho:=a_{1} b_{1}\left(\frac{\gamma}{t_{0}}\right)^{\alpha / \beta} \int_{M} \Phi_{2}\left(a_{1} b_{3} d(y, 0) t_{0}^{-1 / \beta}\right) \varphi(y) d \mu(y),
$$

proving our claim. 
Consider $v(t, x) \equiv u\left(t+t_{0}, x\right)$ such that $u\left(t_{0}, x\right) \geqq 0$. Applying (2.26), we obtain

$$
\begin{aligned}
v(t, x) & \geq \int_{M} k(t, x, y) u\left(t_{0}, y\right) d \mu(y) \\
& \geq \rho \int_{M} k(t, x, y) k(\gamma, y, 0) d \mu(y) \\
& =\rho k(t+\gamma, x, 0),
\end{aligned}
$$

which yields that, using (1.7), $(k 5)$ and Fubini's theorem,

$$
\begin{aligned}
\int_{M} v(t, x) d \mu(x) & \geq \int_{M} d \mu(x) \int_{0}^{t} d \tau \int_{M} k(t-\tau, x, y) v^{p}(\tau, y) d \mu(y) \\
& =\int_{0}^{t} d \tau \int_{M} v^{p}(\tau, y) d \mu(y) \\
& \geq \rho^{p} \int_{0}^{t} d \tau \int_{M} k^{p}(\tau+\gamma, y, 0) d \mu(y) .
\end{aligned}
$$

As $p=1+\beta / \alpha$, we see from (2.3) and $(k 6)$ that

$$
\begin{aligned}
k^{p}(\tau+\gamma, y, 0) & \geq(\tau+\gamma)^{-(1+\alpha / \beta)} \Phi_{1}^{p}\left(d(y, 0)(\tau+\gamma)^{-1 / \beta}\right) \\
& \geq c_{1}(\tau+\gamma)^{-(1+\alpha / \beta)} \Phi_{2}\left(c_{2} d(y, 0)(\tau+\gamma)^{-1 / \beta}\right) \\
& =c_{1} c_{2}^{-\alpha}(\tau+\gamma)^{-1}\left[c_{2}^{-\beta}(\tau+\gamma)\right]^{-\alpha / \beta} \Phi_{2}\left(c_{2} d(y, 0)(\tau+\gamma)^{-1 / \beta}\right) \\
& \geq c_{1} c_{2}^{-\alpha}(\tau+\gamma)^{-1} k\left(c_{2}^{-\beta}(\tau+\gamma), y, 0\right),
\end{aligned}
$$

which combines with (2.27) to give that

$$
\int_{M} v(t, x) d \mu(x) \geq c_{1} c_{2}^{-\alpha} \rho^{p} \int_{0}^{t}(\tau+\gamma)^{-1} d \tau
$$

Passing to the limit as $t \rightarrow \infty$, we conclude that

$$
\int_{M} v(t, x) d \mu(x) \rightarrow \infty
$$

which contradicts (2.25).

We note that our results agree with the earlier ones where $M=\mathbb{R}^{n}$ and $\mu$ is Lebesgue measure, and where the heat kernel $k$ is the Gauss-Weierstrass function (so that $\Delta$ is the usual Laplacian), see [10, 15, 16, 1]. See also [9] where $M$ is a fractal and $\mu$ is $\alpha$-dimenisonal Hausdorff measure, and where $k$ is the Gauss-type heat kernel on $M$.

\section{EXISTENCE OF SOLUTIONS}

In this section we give sufficient conditions for local existence and global existence of weak solutions. 
Theorem 3.1 (Local-existence). Suppose that the heat kernel $k$ satisfies $(k 6)$. Let $b(t)$ be a continuously differentiable function on $\left[0, T_{0}\right)$ satisfying

$$
b^{\prime}(t)=b^{p}(t)\left[\int_{0}^{t} \frac{\left\|K_{\tau} f\right\|_{\infty}}{b(\tau)} d \tau+\left\|K_{t} \varphi\right\|_{\infty}\right]^{p-1}
$$

with initial value $b(0)=1$. If

$$
\int_{0}^{T_{0}}\left[\int_{0}^{s} \frac{\left\|K_{\tau} f\right\|_{\infty}}{b(\tau)} d \tau+\left\|K_{s} \varphi\right\|_{\infty}\right]^{p-1} d s \leq \frac{1}{p-1},
$$

then (1.1)-(1.2) has a non-negative local solution $u \in L^{\infty}((0, T), M)$ for all $0<T<T_{0}$, provided that $\|\varphi\|_{\infty}<\infty$.

Remark 3.2. By Peano's theorem, there exists some $T_{0}>0$ and some continuous differentiable function $b(t)$ such that (3.1) holds in $\left[0, T_{0}\right)$. Clearly, such a $b(t)$ is non-decreasing in $\left[0, T_{0}\right)$. On the other hand, condition (3.2) may be verified for some specific cases. For example, if $(k 5)$ holds and if $f=0, \varphi=C>0$, then

$$
b(t)=\left[1-(p-1) C^{p-1} t\right]^{-1 /(p-1)}
$$

satisfies (3.1) in $\left[0, T_{0}\right)$ where $T_{0}=(p-1)^{-1} C^{-(p-1)}$, and (3.2) also holds. As an another example, let $f=1, \varphi=0$ and assume $(k 5)$ holds. Then, for $p=2$, we see that $b(t)=1 / \cos t$ satisfies (3.1) for $t \in[0, \pi / 2)$, and that (3.2) holds.

Proof. Define

$$
a(t)=b(t) \int_{0}^{t} \frac{\left\|K_{\tau} f\right\|_{\infty}}{b(\tau)} d \tau .
$$

Note that $a(0)=0$ and $a(t) \geq 0$ for $t \in\left[0, T_{0}\right)$. Incorporating this into (3.1), we get

$$
b^{\prime}(t)=b(t)\left[a(t)+b(t)\left\|K_{t} \varphi\right\|_{\infty}\right]^{p-1} .
$$

Moreover,

$$
\begin{aligned}
a^{\prime}(t) & =\left\|K_{t} f\right\|_{\infty}+\frac{b^{\prime}(t) a(t)}{b(t)} \\
& =\left\|K_{t} f\right\|_{\infty}+a(t)\left[a(t)+b(t)\left\|K_{t} \varphi\right\|_{\infty}\right]^{p-1} .
\end{aligned}
$$

Together with the initial conditions, these differential equations are equivalent to

$$
\begin{aligned}
& a(t)=\int_{0}^{t}\left\|K_{\tau} f\right\|_{\infty} d \tau+\int_{0}^{t} a(\tau)\left(a(\tau)+b(\tau)\left\|K_{\tau} \varphi\right\|_{\infty}\right)^{p-1} d \tau, \\
& b(t)=1+\int_{0}^{t} b(\tau)\left(a(\tau)+b(\tau)\left\|K_{\tau} \varphi\right\|_{\infty}\right)^{p-1} d \tau .
\end{aligned}
$$

Let $\mathcal{H}$ be the family of continuous functions $u$ satisfying

$$
K_{t} \varphi(x) \leq u(t, x) \leq a(t)+b(t) K_{t} \varphi(x) \text { for all }(t, x) \in\left[0, T_{0}\right) \times M .
$$


Define

$$
\mathcal{F} u(t, x)=K_{t} \varphi(x)+\int_{0}^{t} K_{\tau} f(x) d \tau+\int_{0}^{t} K_{t-\tau} u^{p}(\tau, x) d \tau .
$$

We claim that if $u \in \mathcal{H}$, then $\mathcal{F} u \in \mathcal{H}$, that is,

$$
K_{t} \varphi(x) \leq \mathcal{F} u(t, x) \leq a(t)+b(t) K_{t} \varphi(x) \quad\left(0 \leq t<T_{0}, x \in M\right) .
$$

Observe that, using $(k 1)$,

$$
\begin{aligned}
\int_{0}^{t} K_{t-\tau}\left[a(\tau)+b(\tau) K_{\tau} \varphi\right]^{p}(x) d \tau \\
\quad=\int_{0}^{t} d \tau \int_{M} k(t-\tau, x, y)\left[a(\tau)+b(\tau) K_{\tau} \varphi(y)\right]^{p} d \mu(y) \\
\quad \leq \int_{0}^{t}\left[a(\tau)+b(\tau)\left\|K_{\tau} \varphi\right\|_{\infty}\right]^{p-1}\left[a(\tau)+b(\tau) K_{t} \varphi(x)\right] d \tau .
\end{aligned}
$$

It follows from (3.6) and (3.5) that

$$
\begin{aligned}
\mathcal{F} u(t, x) \leq & K_{t} \varphi(x)+\int_{0}^{t} K_{\tau} f(x) d \tau+\int_{0}^{t} K_{t-\tau}\left[a(\tau)+b(\tau) K_{\tau} \varphi\right]^{p}(x) d \tau \\
\leq & {\left[\int_{0}^{t}\left\|K_{\tau} f\right\|_{\infty} d \tau+\int_{0}^{t} a(\tau)\left[a(\tau)+b(\tau)\left\|K_{\tau} \varphi\right\|_{\infty}\right]^{p-1} d \tau\right] } \\
& +\left[1+\int_{0}^{t} b(\tau)\left[a(\tau)+b(\tau)\left\|K_{\tau} \varphi\right\|_{\infty}\right]^{p-1} d \tau\right] K_{t} \varphi(x) \\
= & a(t)+b(t) K_{t} \varphi(x)
\end{aligned}
$$

using (3.3) and (3.4), so (3.7) holds, proving our claim.

For $n=0,1,2, \cdots$, define

$$
\begin{aligned}
u_{0}(t, x) & =K_{t} \varphi(x), \\
u_{n+1}(t, x) & =\mathcal{F} u_{n}(t, x) .
\end{aligned}
$$

Using (3.6) inductively, it follows that the sequence $\left\{u_{n}(t, x)\right\}$ is non-decreasing in $n$, and, for all $n \geq 0$ and all $x \in M, t \in\left[0, T_{0}\right.$ ), satisfies

$$
K_{t} \varphi(x) \leq u_{n}(t, x) \leq a(t)+b(t) K_{t} \varphi(x) .
$$

Let $u(t, x):=\lim _{n \rightarrow \infty} u_{n}(t, x)$. Note that $K_{t} \varphi(x) \leq u(t, x) \leq a(t)+b(t) K_{t} \varphi(x)$.

Using the monotone convergence theorem, we have

$$
\lim _{n \rightarrow \infty} \int_{0}^{t} d \tau \int_{M} k(t-\tau, x, y) u_{n}^{p}(\tau, y) d \mu(y)=\int_{0}^{t} d \tau \int_{M} k(t-\tau, x, y) u^{p}(\tau, y) d \mu(y) .
$$

Since $u_{n}(t, x)$ satisfies

$$
u_{n+1}(t, x)=K_{t} \varphi(x)+\int_{0}^{t} K_{\tau} f(x) d \tau+\int_{0}^{t} K_{t-\tau} u_{n}^{p}(\tau, x) d \tau,
$$

we pass to the limit as $n \rightarrow \infty$ to obtain

$$
u(t, x)=K_{t} \varphi(x)+\int_{0}^{t} K_{\tau} f(x) d \tau+\int_{0}^{t} K_{t-\tau} u^{p}(\tau, x) d \tau,
$$


which shows that $u(t, x)$ is a non-negative local solution of (1.1)-(1.2) for $t \in\left[0, T_{0}\right)$.

Since $a(t), b(t)$ are differentiable functions on $\left[0, T_{0}\right)$, we see from (3.5) that for all $t \in\left[0, T_{0}\right)$,

$$
\|u(t, \cdot)\|_{\infty} \leq\left\|a(t)+b(t) K_{t} \varphi\right\|_{\infty}<\infty .
$$

The proof is complete.

Recall that, by Theorem 2.3, (1.1)-(1.2) does not have any essentially bounded global weak solution if $\alpha>\beta$ and $p<\frac{\alpha}{\alpha-\beta}$ and if $f(x) \geqq 0$. However, we can show that (1.1)-(1.2) possesses a essentially bounded global solution if $p>\frac{\alpha}{\alpha-\beta}$, for small functions $f$ and $\varphi$ (cf. [17] for Euclidean spaces). To do this, we need some integral estimates which are consequences of measure bounds for small and large balls.

Recall that a measure $\mu$ on a metric measure space is upper $\alpha$-regular if there exist some $C, \alpha>0$ such that

$$
\left.\mu(B(x, r)) \leq C r^{\alpha} \text { (for all } x \in M, r>0\right),
$$

and is $\alpha$-regular if there exists a constant $C>0$ such that for all $x \in M$ and all $r>0$,

$$
\left.C^{-1} r^{\alpha} \leq \mu(B(x, r)) \leq C r^{\alpha} \text { (for all } x \in M, r>0\right) .
$$

It was shown in [11, Theorem 3.2] that if the heat kernel $k$ satisfies $(k 5),(k 6)$ with $\Phi_{2}(s)$ satisfying

$$
\int_{0}^{\infty} s^{\alpha-1} \Phi_{2}(s) d s<\infty
$$

then the measure $\mu$ is $\alpha$-regular. Note that, by the monotonicity of $\Phi_{2}$, condition (3.11) implies that $s^{\alpha} \Phi_{2}(s) \leq C<\infty$ for all $s \in[0, \infty)$.

Proposition 3.3. Assume that $\mu$ is upper $\alpha$-regular and $x_{0}$ is a reference point in $M$. If $0<\lambda_{1}<\alpha$ and $\lambda_{1}+\lambda_{2}>\alpha$, then there exists a constant $C_{0}>0$ such that

$$
\int_{M} \frac{1}{d(y, x)^{\lambda_{1}}\left[1+d\left(y, x_{0}\right)^{\lambda_{2}}\right]} d \mu(y) \leq C_{0} \quad(\text { for all } x \in M) .
$$

Proof. For each $x \in M$, let $\Omega_{1}=\left\{y \in M: d(y, x) \geq d\left(y, x_{0}\right)\right\}$ and $\Omega_{2}=$ $M \backslash \Omega_{1}$. Then

$$
\int_{\Omega_{1}} \frac{1}{d(y, x)^{\lambda_{1}}\left[1+d\left(y, x_{0}\right)^{\lambda_{2}}\right]} d \mu(y) \leq \int_{M} \frac{1}{d\left(y, x_{0}\right)^{\lambda_{1}}\left[1+d\left(y, x_{0}\right)^{\lambda_{2}}\right]} d \mu(y)
$$

and

$$
\int_{\Omega_{2}} \frac{1}{d(y, x)^{\lambda_{1}}\left[1+d\left(y, x_{0}\right)^{\lambda_{2}}\right]} d \mu(y) \leq \int_{M} \frac{1}{d(y, x)^{\lambda_{1}}\left[1+d(y, x)^{\lambda_{2}}\right]} d \mu(y) .
$$

Routine estimates using upper regularity (3.9) now give uniform bounds on these integrals near $x_{0}$ and $x$ (since $\lambda_{1}<\alpha$ ) and for large $d\left(y, x_{0}\right)$ and $d(y, x)$ (since $\lambda_{1}+\lambda_{2}>\alpha$ ), to give (3.12). 
Proposition 3.4. Assume that $\mu$ is upper $\alpha$-regular and $x_{0}$ is a reference point in $M$. If $0<\lambda_{1}<\alpha$ and $\lambda_{2}>\alpha$, then there exists a constant $C_{1}>0$ such that

$$
\int_{M} \frac{1}{d(y, x)^{\lambda_{1}}\left[1+d\left(y, x_{0}\right)^{\lambda_{2}}\right]} d \mu(y) \leq \frac{C_{1}}{1+d\left(x, x_{0}\right)^{\lambda_{1}}} .
$$

Proof. Fix $x \in M$. If $d\left(x, x_{0}\right) \leq 1$, then (3.13) directly follows from (3.12), since

$$
\int_{M} \frac{1}{d(y, x)^{\lambda_{1}}\left[1+d\left(y, x_{0}\right)^{\lambda_{2}}\right]} d \mu(y) \leq C_{0} \leq \frac{2 C_{0}}{1+d\left(x, x_{0}\right)^{\lambda_{1}}} .
$$

Assume that $d\left(x, x_{0}\right) \geq 1$. If $d\left(y, x_{0}\right) \geq d\left(x, x_{0}\right) / 2$, we have that

$$
\frac{1}{1+d\left(y, x_{0}\right)^{\lambda_{2}}} \leq \frac{C}{\left[1+d\left(y, x_{0}\right)^{\lambda_{2}-\lambda_{1}}\right]\left[1+d\left(x, x_{0}\right)^{\lambda_{1}}\right]}
$$

where $C$ is independent of $x_{0}, y$. Using Proposition 3.3 , it follows that

$$
\begin{aligned}
& \int_{d\left(y, x_{0}\right) \geq d\left(x, x_{0}\right) / 2} \frac{1}{d(y, x)^{\lambda_{1}}\left[1+d\left(y, x_{0}\right)^{\lambda_{2}}\right]} d \mu(y) \\
\leq & \frac{C}{1+d\left(x, x_{0}\right)^{\lambda_{1}}} \int_{d\left(y, x_{0}\right) \geq d\left(x, x_{0}\right) / 2} \frac{1}{d(y, x)^{\lambda_{1}}\left[1+d\left(y, x_{0}\right)^{\lambda_{2}-\lambda_{1}}\right]} d \mu(y) \\
\leq & \frac{C_{1}}{1+d\left(x, x_{0}\right)^{\lambda_{1}}} .
\end{aligned}
$$

If $d\left(y, x_{0}\right)<d\left(x, x_{0}\right) / 2$, then

$$
d(y, x)^{-\lambda_{1}} \leq\left[d\left(x, x_{0}\right)-d\left(y, x_{0}\right)\right]^{-\lambda_{1}} \leq\left[d\left(x, x_{0}\right) / 2\right]^{-\lambda_{1}} \leq \frac{2^{\lambda_{1}+1}}{1+d\left(x, x_{0}\right)^{\lambda_{1}}},
$$

and hence,

$$
\int_{d\left(y, x_{0}\right)<d\left(x, x_{0}\right) / 2} \frac{1}{d(y, x)^{\lambda_{1}}\left[1+d\left(y, x_{0}\right)^{\lambda_{2}}\right]} d \mu(y) \leq \frac{C_{2}}{1+d\left(x, x_{0}\right)^{\lambda_{1}}} .
$$

where we have used that $\int_{M} \frac{1}{1+d\left(y, x_{0}\right)^{\lambda_{2}}} d \mu(y)<\infty$ as $\lambda_{2}>\alpha$.

Adding (3.14) and (3.16) we see that (3.13) also holds if $d\left(x, x_{0}\right) \geq 1$.

We now show the global existence of weak solutions for small $\varphi$ and $f$.

Theorem 3.5 (Global-existence). Let $\alpha>\beta>0$ and suppose that the heat kernel $k$ satisfies $(k 5),(k 6)$ and that $\Phi_{2}$ satisfies (3.11). Let $\lambda>\alpha$ and let $x_{0}$ be a reference point in $M$. The for each $p>\alpha /(\alpha-\beta)$ there exists $\delta>0$ such that if

$$
0<\varphi(x), f(x) \leq \frac{\delta}{1+d\left(x, x_{0}\right)^{\lambda}}
$$

then (1.1)-(1.2) has an essentially bounded global solution. 
Proof. Recall that conditions $(k 5),(k 6)$ and (3.11) imply that $\mu$ is $\alpha$ regular. Let the map $\mathcal{F}$ be defined as in (3.6):

$$
\mathcal{F} u(t, x)=K_{t} \varphi(x)+\int_{0}^{t} K_{\tau} f(x) d \tau+\int_{0}^{t} K_{t-\tau} u^{p}(\tau, x) d \tau .
$$

For $\epsilon>0$, let $S_{\varepsilon}$ be the complete subset of the Banach space $L^{\infty}([0, \infty) \times M)$ given by

$$
S_{\varepsilon}=\left\{u \in L^{\infty}([0, \infty) \times M): 0 \leq u(t, x) \leq \frac{\varepsilon}{1+d\left(x, x_{0}\right)^{\alpha-\beta}}\right\}
$$

We will use the contraction principle to show that, for appropriately small $\epsilon$ and $\delta$, there exists a global solution in $S_{\varepsilon}$.

For $\lambda>\alpha$, we claim that there exists $C_{2}>0$ such that, for all $0 \leq g(x) \leq$ $\delta /\left(1+d\left(x, x_{0}\right)^{\lambda}\right)$, we have

$$
K_{t} g(x) \leq \frac{C_{2} \delta}{1+d\left(x, x_{0}\right)^{\alpha}} \text { for all } x \in M \text { and all } t>0 .
$$

To see this, let $x \in M$. If $d\left(x, x_{0}\right) \leq 1$, then (3.17) is clear since

$$
\begin{aligned}
K_{t} g(x) & =\int_{M} k(t, x, y) g(y) d \mu(y) \\
& \leq \int_{M} \frac{\delta}{1+d\left(y, x_{0}\right)^{\lambda}} k(t, x, y) d \mu(y) \\
& \leq \delta \int_{M} k(t, x, y) d \mu(y) \leq \delta \\
& \leq \frac{2 \delta}{1+d\left(x, x_{0}\right)^{\alpha}} .
\end{aligned}
$$

So assume $d\left(x, x_{0}\right)>1$. We have, using condition $(k 6)$,

$$
\begin{aligned}
K_{t} g(x) \leq & \int_{M} \frac{\delta}{1+d\left(y, x_{0}\right)^{\lambda}} k(t, x, y) d \mu(y) \\
\leq & \delta\left\{\int_{\Omega_{1}} \frac{1}{1+d\left(y, x_{0}\right)^{\lambda}} \frac{1}{t^{\alpha / \beta}} \Phi_{2}\left(\frac{d(y, x)}{t^{1 / \beta}}\right) d \mu(y)\right. \\
& \left.+\int_{\Omega_{2}} \frac{1}{1+d\left(y, x_{0}\right)^{\lambda}} k(t, x, y) d \mu(y)\right\},
\end{aligned}
$$

where $\Omega_{1}=\left\{y \in M: d\left(y, x_{0}\right) \leq d\left(x, x_{0}\right) / 2\right\}$ and $\Omega_{2}=M \backslash \Omega_{1}$. For $y \in \Omega_{1}$, we have, noting from (3.11) that $s^{\alpha} \Phi_{2}(s)$ is bounded,

$$
\begin{aligned}
\frac{1}{t^{\alpha / \beta}} \Phi_{2}\left(\frac{d(y, x)}{t^{1 / \beta}}\right) & =\frac{1}{d(y, x)^{\alpha}}\left(\frac{d(y, x)}{t^{1 / \beta}}\right)^{\alpha} \Phi_{2}\left(\frac{d(y, x)}{t^{1 / \beta}}\right) \\
& \leq \frac{C}{d(y, x)^{\alpha}} \leq \frac{2^{\alpha} C}{d\left(x, x_{0}\right)^{\alpha}} \\
& \leq \frac{2^{\alpha+1} C}{1+d\left(x, x_{0}\right)^{\alpha}}
\end{aligned}
$$


and hence, using that $\int_{M} \frac{d \mu(y)}{1+d\left(y, x_{0}\right)^{\lambda}}<+\infty$ for $\lambda>\alpha$,

$$
\begin{aligned}
\int_{\Omega_{1}} \frac{1}{1+d\left(y, x_{0}\right)^{\lambda}} \frac{1}{t^{\alpha / \beta}} \Phi_{2}\left(\frac{d(y, x)}{t^{1 / \beta}}\right) d \mu(y) & \leq \frac{2^{\alpha+1} C}{1+d\left(x, x_{0}\right)^{\alpha}} \int_{\Omega_{1}} \frac{d \mu(y)}{1+d\left(y, x_{0}\right)^{\lambda}} \\
& \leq \frac{C}{1+d\left(x, x_{0}\right)^{\alpha}}
\end{aligned}
$$

For $y \in \Omega_{2}$,

$$
\begin{aligned}
\int_{\Omega_{2}} \frac{1}{1+d\left(y, x_{0}\right)^{\lambda}} k(t, x, y) d \mu(y) & \leq \frac{2^{\lambda}}{1+d\left(x, x_{0}\right)^{\lambda}} \int_{\Omega_{2}} k(t, x, y) d \mu(y) \\
& \leq \frac{C}{1+d\left(x, x_{0}\right)^{\alpha}}
\end{aligned}
$$

using that $\lambda>\alpha$. Adding (3.19) and (3.20), we see that (3.17) follows from (3.18), proving our claim.

Observe that by $(k 6)$ and (3.11),

$$
\begin{aligned}
\int_{0}^{t} k(\tau, x, y) d \tau & \leq \int_{0}^{t} \frac{1}{\tau^{\alpha / \beta}} \Phi_{2}\left(\frac{d(y, x)}{\tau^{1 / \beta}}\right) d \tau \\
& =\frac{\beta}{d(y, x)^{\alpha-\beta}} \int_{d(x, y) / t^{1 / \beta}}^{\infty} s^{\alpha-\beta-1} \Phi_{2}(s) d s \\
& \leq \frac{\beta}{d(y, x)^{\alpha-\beta}} \int_{0}^{\infty} s^{\alpha-\beta-1} \Phi_{2}(s) d s \\
& \leq \frac{C}{d(y, x)^{\alpha-\beta}}
\end{aligned}
$$

since

$$
\int_{0}^{\infty} s^{\alpha-\beta-1} \Phi_{2}(s) d s \leq \Phi_{2}(0) \int_{0}^{1} s^{\alpha-\beta-1} d s+\int_{1}^{\infty} s^{\alpha-1} \Phi_{2}(s) d s<+\infty
$$

using the monotonicity of $\Phi_{2}$ and (3.11).

Therefore, using (3.21) and (3.13) with $\lambda_{1}=\alpha-\beta>0$ and $\lambda_{2}=\lambda>\alpha$,

$$
\begin{aligned}
\int_{0}^{t} K_{\tau} f(x) d \tau & =\int_{M}\left[\int_{0}^{t} k(\tau, x, y) d \tau\right] f(y) d \mu(y) \\
& \leq \int_{M} \frac{C}{d(y, x)^{\alpha-\beta}} \frac{\delta}{\left(1+d\left(y, x_{0}\right)^{\lambda}\right)} d \mu(y) \\
& \leq \frac{C \delta}{1+d\left(x, x_{0}\right)^{\alpha-\beta}}
\end{aligned}
$$


for all $x \in M$ and $t>0$. Similarly, for $u \in S_{\varepsilon}$, we have that, using (3.13) with $\lambda_{1}=\alpha-\beta, \lambda_{2}=p(\alpha-\beta)>\alpha$,

$$
\begin{aligned}
\int_{0}^{t} K_{t-\tau} u^{p}(\tau, x) d \tau & \leq \int_{0}^{t} \int_{M} k(t-\tau, x, y) \frac{\varepsilon^{p}}{\left(1+d\left(y, x_{0}\right)^{\alpha-\beta}\right)^{p}} d \mu(y) d \tau \\
& \leq \int_{M} \frac{C}{d(y, x)^{\alpha-\beta}} \frac{\varepsilon^{p}}{\left(1+d\left(y, x_{0}\right)^{\alpha-\beta}\right)^{p}} d \mu(y) \\
& \leq C \varepsilon^{p} \int_{M} \frac{1}{d(y, x)^{\alpha-\beta}} \frac{1}{1+d\left(y, x_{0}\right)^{(\alpha-\beta) p}} d \mu(y) \\
& \leq \frac{C \varepsilon^{p}}{1+d\left(x, x_{0}\right)^{\alpha-\beta}}
\end{aligned}
$$

for all $x \in M$ and $t>0$. It follows from (3.17), (3.22), (3.23) that if $u \in S_{\varepsilon}$, then

$$
\begin{aligned}
\mathcal{F} u(t, x) & \leq \frac{C_{2} \delta}{1+d\left(x, x_{0}\right)^{\alpha}}+\frac{C \delta+C \varepsilon^{p}}{1+d\left(x, x_{0}\right)^{\alpha-\beta}} \\
& \leq \frac{C_{1}\left(\delta+\varepsilon^{p}\right)}{1+d\left(x, x_{0}\right)^{\alpha-\beta}} \\
& \leq \frac{\varepsilon}{1+d\left(x, x_{0}\right)^{\alpha-\beta}}
\end{aligned}
$$

provided that $C_{1}\left(\delta+\varepsilon^{p}\right) \leq \varepsilon$, in which case $\mathcal{F} S_{\varepsilon} \subset S_{\varepsilon}$.

Next we show that $\mathcal{F}$ is contractive on $S_{\varepsilon}$. Indeed, for $u_{1}, u_{2} \in S_{\varepsilon}$, we have

$$
\left|\mathcal{F} u_{1}(t, x)-\mathcal{F} u_{2}(t, x)\right| \leq \int_{0}^{t} \int_{M} k(t-\tau, x, y)\left|u_{1}^{p}(\tau, y)-u_{2}^{p}(\tau, y)\right| d \mu(y) d \tau .
$$

Using the elementary inequality

$$
\left|a^{p}-b^{p}\right| \leq p \max \left\{a^{p-1}, b^{p-1}\right\}|a-b| \text { for } a, b \geq 0, p>1,
$$

and the definition of $S_{\varepsilon}$, we obtain, using ( $(3.21)$ and (3.12), that

$$
\begin{aligned}
& \left|\mathcal{F} u_{1}(t, x)-\mathcal{F} u_{2}(t, x)\right| \\
& \quad \leq\left\|u_{1}-u_{2}\right\|_{\infty} \int_{0}^{t} \int_{M} k(t-\tau, x, y) \frac{p \varepsilon^{p-1}}{\left[1+d\left(y, x_{0}\right)^{\alpha-\beta}\right]^{p-1}} d \mu(y) d \tau \\
& \quad \leq\left\|u_{1}-u_{2}\right\|_{\infty} \int_{M} \frac{C}{d(y, x)^{\alpha-\beta}} \frac{p \varepsilon^{p-1}}{1+d\left(y, x_{0}\right)^{(\alpha-\beta)(p-1)}} d \mu(y) \\
& \quad \leq C_{3} p \varepsilon^{p-1}\left\|u_{1}-u_{2}\right\|_{\infty} .
\end{aligned}
$$

Thus if $\epsilon$ is small enough to ensure that both $C_{3} p \varepsilon^{p-1}<1$ and $C_{1} \varepsilon^{p}<\varepsilon$, and then $\delta$ is chosen small enough so that $C_{1}\left(\delta+\varepsilon^{p}\right) \leq \varepsilon$, applying Banach's contraction principle to $\mathcal{F}$ on the complete set $S_{\varepsilon}$ implies that (1.7) and thus (1.1)-(1.2) has a global positive solution in $S_{\varepsilon}$. 


\section{Regularity}

In this section, we discuss the regularity of weak solutions. We show that weak solutions are Hölder continuous in the spatial variable $x$ if the source term $f$ and initial value $\varphi$ are both Hölder continuous. We adapt the method used in [9].

In order to obtain the regularity of weak solutions, we need to assume that the function $\Phi_{2}$ in condition $(k 6)$ satisfies the following assumption:

$$
\int_{0}^{\infty} s^{\alpha} \Phi_{2}(s) d s<\infty
$$

where $\alpha$ is as in condition $(k 6)$. Since $\Phi_{2}$ is non-increasing on $[0, \infty)$, condition (4.1) implies that $s^{1+\alpha} \Phi_{2}(s)=o(1)$ as $s \rightarrow \infty$.

Clearly, the Gauss-type function $\Phi_{2}$ defined as in (2.4) satisfies condition (4.1) for all $\gamma>0$ whilst the Cauchy-type function $\Phi_{2}$ defined as in (2.5) satisfies condition (4.1) for all $\gamma>1+\alpha$.

Note that condition (4.1) is stronger than (3.11), and hence it implies that $\mu$ is $\alpha$-regular.

Proposition 4.1. Assume that $\mu$ is upper $\alpha$-regular. If $\Phi_{2}$ satisfies (4.1) then, for all $\lambda \in(0,1]$,

$$
\int_{M} d(x, y)^{\lambda} \Phi_{2}\left(\frac{d(x, y)}{t^{1 / \beta}}\right) d \mu(y) \leq C_{2} t^{(\alpha+\lambda) / \beta} \quad(\text { for all } x \in M, t>0)
$$

for some constant $C_{2}$.

Proof. Let $g(r)=r^{\lambda} \Phi_{2}\left(\frac{r}{t^{1 / \beta}}\right)$ for $r>0$. From (4.2) $g(r)=o\left(r^{-\alpha}\right)$ so, by a standard argument using $\alpha$-regularity and integration by parts (see [9. Proposition 4.1]), it follows that

$$
\begin{aligned}
\int_{M} d(x, y)^{\lambda} & \Phi_{2}\left(\frac{d(x, y)}{t^{1 / \beta}}\right) d \mu(y)=\int_{M} g(d(x, y)) d \mu(y) \\
& \leq C_{1} \int_{0}^{\infty} r^{\alpha}\left|g^{\prime}(r)\right| d r \\
& =C_{1} \int_{0}^{\infty} r^{\alpha}\left|\lambda r^{\lambda-1} \Phi_{2}\left(\frac{r}{t^{1 / \beta}}\right)+r^{\lambda} \Phi_{2}^{\prime}\left(\frac{r}{t^{1 / \beta}}\right) t^{-1 / \beta}\right| d r \\
& \leq C_{2} t^{(\alpha+\lambda) / \beta}\left[\int_{0}^{\infty} \lambda s^{\alpha+\lambda-1} \Phi_{2}(s) d s+\int_{0}^{\infty} s^{\alpha+\lambda}\left(-\Phi_{2}^{\prime}(s)\right) d s\right] .
\end{aligned}
$$

By an easy calculation, the last integral

$$
\begin{aligned}
\int_{0}^{\infty} s^{\alpha+\lambda}\left(-\Phi_{2}^{\prime}(s)\right) d s & =-\left.s^{\alpha+\lambda} \Phi_{2}(s)\right|_{0} ^{\infty}+(\alpha+\lambda) \int_{0}^{\infty} s^{\alpha+\lambda-1} \Phi_{2}(s) d s \\
& =(\alpha+\lambda) \int_{0}^{\infty} s^{\alpha+\lambda-1} \Phi_{2}(s) d s \leq C_{3}
\end{aligned}
$$

using (4.1). Therefore,

$$
\int_{M} d(x, y)^{\lambda} \Phi\left(\frac{d(x, y)}{t^{1 / \beta}}\right) d \mu(y) \leq C_{2} t^{(\alpha+\lambda) / \beta},
$$


as desired.

We now show the Hölder continuity of weak solutions of (1.7).

Theorem 4.2 (Hölder Continuity). Assume that $\varphi, f \in L^{1}(M)$ are Hölder continuous with exponents $\theta_{1}, \theta_{2} \in(0,1]$ respectively: for all $x_{1}, x_{2} \in M$,

$$
\begin{aligned}
\left|\varphi\left(x_{1}\right)-\varphi\left(x_{2}\right)\right| & \leq C_{5} d\left(x_{1}, x_{2}\right)^{\theta_{1}} \\
\left|f\left(x_{1}\right)-f\left(x_{2}\right)\right| & \leq C_{6} d\left(x_{1}, x_{2}\right)^{\theta_{2}}
\end{aligned}
$$

where $C_{5}, C_{6}>0$. Assume that the heat kernel $k$ satisfies $(k 5)-(k 7)$ and that $\Phi_{2}$ satisfies (4.1) with $\lambda=\max \left\{\theta_{1}, \theta_{2}\right\}$. Let $u(t, x)$ be a non-negative weak solution to (1.1)-(1.2) that is bounded in $(0, T) \times M$ for some $T>0$. Then $u(t, x)$ is Hölder continuous: for all $x_{1}, x_{2} \in M$ and all $t \in(0, T)$,

$$
\left|u\left(t, x_{1}\right)-u\left(t, x_{2}\right)\right| \leq C d\left(x_{1}, x_{2}\right)^{\theta}
$$

where $\theta=\theta_{1} \sigma /\left(\theta_{1}+v \beta\right)$ and $C>0$ may depend on $T$ but is independent of $t, x$.

Proof. From ( $k 6)$, (4.3), and (4.2), there exists $C>0$ such that for all $t>0$ and $x \in M$,

$$
\begin{aligned}
\int_{M} k(t, x, y)|\varphi(y)-\varphi(x)| d \mu(y) & \leq C_{5} t^{-\alpha / \beta} \int_{M} d(x, y)^{\theta_{1}} \Phi_{2}\left(\frac{d(x, y)}{t^{1 / \beta}}\right) d \mu(y) \\
& \leq C t^{\theta_{1} / \beta}
\end{aligned}
$$

By (1.7) it is enough to show that each of the functions $u_{0}, u_{1}, u_{2}$ is Hölder continuous in $(0, T) \times M$, where

$$
\begin{aligned}
& u_{0}(t, x)=K_{t} \varphi(x), \\
& u_{1}(t, x)=\int_{0}^{t} K_{\tau} f(x) d \tau, \\
& u_{2}(t, x)=\int_{0}^{t} K_{t-\tau} u^{p}(\tau, x) d \tau .
\end{aligned}
$$

We first show the Hölder continuity of $u_{0}$. Indeed, for $t>0$ and $x_{1}, x_{2} \in M$, we see from $(k 7)$ that

$$
\begin{aligned}
\left|u_{0}\left(t, x_{1}\right)-u_{0}\left(t, x_{2}\right)\right| & =\left|\int_{M}\left(k\left(t, x_{1}, y\right)-k\left(t, x_{2}, y\right)\right) \varphi(y) d \mu(y)\right| \\
& \leq L t^{-v} d\left(x_{1}, x_{2}\right)^{\sigma}\|\varphi\|_{1} \\
& \leq L\|\varphi\|_{1} d\left(x_{1}, x_{2}\right)^{\sigma-v s_{0}}
\end{aligned}
$$


if $t \geq d\left(x_{1}, x_{2}\right)^{s_{0}}$, where $s_{0}>0$ will be specified later on. On the other hand, if $t \leq d\left(x_{1}, x_{2}\right)^{s_{0}}$, we have, using (k5), (4.6) and (4.3), that

$$
\begin{aligned}
\left|u_{0}\left(t, x_{1}\right)-u_{0}\left(t, x_{2}\right)\right| \leq & \mid \int_{M} k\left(t, x_{1}, y\right)\left(\varphi(y)-\varphi\left(x_{1}\right)\right) d \mu(y) \\
& +\left[\varphi\left(x_{1}\right)-\varphi\left(x_{2}\right)\right]-\int_{M} k\left(t, x_{2}, y\right)\left(\varphi(y)-\varphi\left(x_{2}\right)\right) d \mu(y) \mid \\
\leq & 2 C t^{\theta_{1} / \beta}+C_{5} d\left(x_{1}, x_{2}\right)^{\theta_{1}} \\
\leq & C\left[d\left(x_{1}, x_{2}\right)^{s_{0} \theta_{1} / \beta}+d\left(x_{1}, x_{2}\right)^{\theta_{1}}\right] .
\end{aligned}
$$

Combining this with (4.7), it follows that

$$
\begin{aligned}
\left|u_{0}\left(t, x_{1}\right)-u_{0}\left(t, x_{2}\right)\right| & \leq C\left[d\left(x_{1}, x_{2}\right)^{\sigma-v s_{0}}+d\left(x_{1}, x_{2}\right)^{s_{0} \theta_{1} / \beta}+d\left(x_{1}, x_{2}\right)^{\theta_{1}}\right] \\
& \leq C d\left(x_{1}, x_{2}\right)^{\theta_{1} \sigma /\left(\theta_{1}+v \beta\right)}
\end{aligned}
$$

for all $t>0$ and $x_{1}, x_{2} \in M$ with $d\left(x_{1}, x_{2}\right) \leq 1$, where $s_{0}=\sigma /\left(v+\frac{\theta_{1}}{\beta}\right)$ so that $\sigma-v s_{0}=s_{0} \theta_{1} / \beta$, and where we have used the fact that $\theta_{1} \geq s_{0} \theta_{1} / \beta$ for $\sigma \leq 1 \leq v$ and $\beta \geq 1$.

Next we show the Hölder continuity of $u_{1}$. As with (4.6), we have from $(k 6)$, (4.4) and (4.2) that

$$
\int_{M} k\left(\tau, x_{1}, y\right)\left|f(y)-f\left(x_{1}\right)\right| d \mu(y) \leq C \tau^{\theta_{2} / \beta}
$$

which yields that, using $(k 5)$ and (4.4),

$$
\begin{aligned}
\left|u_{1}\left(t, x_{1}\right)-u_{1}\left(t, x_{2}\right)\right|= & \left|\int_{0}^{t}\left[K_{\tau} f\left(x_{1}\right)-K_{\tau} f\left(x_{2}\right)\right] d \tau\right| \\
= & \mid \int_{0}^{t} d \tau \int_{M} k\left(\tau, x_{1}, y\right)\left(f(y)-f\left(x_{1}\right)\right) d \mu(y) \\
& +t\left[f\left(x_{1}\right)-f\left(x_{2}\right)\right]-\int_{0}^{t} d \tau \int_{M} k\left(\tau, x_{2}, y\right)\left(f(y)-f\left(x_{2}\right)\right) d \mu(y) \mid \\
\leq & 2 C \int_{0}^{t} \tau^{\theta_{2} / \beta} d \tau+C_{6} t d\left(x_{1}, x_{2}\right)^{\theta_{2}} \\
= & C t^{\theta_{2} / \beta+1}+C_{6} t d\left(x_{1}, x_{2}\right)^{\theta_{2}} \\
\leq & C\left[d\left(x_{1}, x_{2}\right)^{s_{1}+s_{1} \theta_{2} / \beta}+d\left(x_{1}, x_{2}\right)^{s_{1}+\theta_{2}}\right]
\end{aligned}
$$

if $t \leq d\left(x_{1}, x_{2}\right)^{s_{1}}$, where $s_{1}>0$ will be chosen later. 
On the other hand, if $t>d\left(x_{1}, x_{2}\right)^{s_{1}}$, and setting $t_{1}=d\left(x_{1}, x_{2}\right)^{s_{1}}$, we obtain, using $(k 7)$, that

$$
\begin{aligned}
\left|\int_{t_{1}}^{t}\left[K_{\tau} f\left(x_{1}\right)-K_{\tau} f\left(x_{2}\right)\right] d \tau\right| & \leq \int_{t_{1}}^{t} d \tau \int_{M}\left|k\left(\tau, x_{1}, y\right)-k\left(\tau, x_{2}, y\right)\right||f(y)| d \mu(y) \\
& \leq \int_{t_{1}}^{t} L \tau^{-v} d\left(x_{1}, x_{2}\right)^{\sigma}\|f\|_{1} d \tau \\
& \leq L \frac{t_{1}^{1-v}-t^{1-v}}{v-1} d\left(x_{1}, x_{2}\right)^{\sigma}\|f\|_{1} \\
& \leq \frac{L}{v-1} d\left(x_{1}, x_{2}\right)^{s_{1}(1-v)+\sigma}\|f\|_{1} .
\end{aligned}
$$

It follows from (4.10) and (4.9) that

$$
\begin{aligned}
\left|u_{1}\left(t, x_{1}\right)-u_{1}\left(t, x_{2}\right)\right| & \leq\left|\int_{0}^{t_{1}} K_{\tau} f\left(x_{1}\right)-K_{\tau} f\left(x_{2}\right) d \tau\right|+\left|\int_{t_{1}}^{t} K_{\tau} f\left(x_{1}\right)-K_{\tau} f\left(x_{2}\right) d \tau\right| \\
& \leq C\left[d\left(x_{1}, x_{2}\right)^{s_{1}+s_{1} \theta_{2} / \beta}+d\left(x_{1}, x_{2}\right)^{s_{1}+\theta_{2}}+d\left(x_{1}, x_{2}\right)^{s_{1}(1-v)+\sigma}\right] \\
& \leq C d\left(x_{1}, x_{2}\right)^{\sigma\left(\theta_{2}+\beta\right) /\left(\theta_{2}+\nu \beta\right)}
\end{aligned}
$$

if $d\left(x_{1}, x_{2}\right) \leq 1$, where $s_{1}=\sigma \beta /\left(\theta_{2}+v \beta\right)$ so that $s_{1}+s_{1} \theta_{2} / \beta=s_{1}(1-v)+\sigma$, and where we have used the fact that

$$
s_{1}+\theta_{2} \geq s_{1}+s_{1} \theta_{2} / \beta
$$

for $\sigma \leq 1 \leq v$ and $\beta \geq 1$.

Finally, we show the Hölder continuity of $u_{2}$. Since $u(t, x)$ is bounded on $(0, T) \times M$, we see that

$$
\int_{t-\eta}^{t} d \tau \int_{M} k(t-\tau, x, y) u^{p}(\tau, y) d \mu(y) \leq C \eta .
$$

Hence, using $(k 7)$, we obtain

$$
\begin{aligned}
\left|u_{2}\left(t, x_{1}\right)-u_{2}\left(t, x_{2}\right)\right|= & \mid \int_{t-\eta}^{t} d \tau \int_{M} k\left(t-\tau, x_{1}, y\right) u^{p}(\tau, y) d \mu(y) \\
& -\int_{t-\eta}^{t} d \tau \int_{M} k\left(t-\tau, x_{2}, y\right) u^{p}(\tau, y) d \mu(y) \\
& +\int_{0}^{t-\eta} d \tau \int_{M}\left(k\left(t-\tau, x_{1}, y\right)-k\left(t-\tau, x_{2}, y\right)\right) u^{p}(\tau, y) d \mu(y) \mid \\
\leq & 2 C \eta+L \int_{0}^{t-\eta} d \tau \int_{M}|t-\tau|^{-v} d\left(x_{1}, x_{2}\right)^{\sigma} u^{p}(\tau, y) d \mu(y) \\
\leq & C\left(\eta+\eta^{1-v} d\left(x_{1}, x_{2}\right)^{\sigma}\right) .
\end{aligned}
$$

Taking $\eta=d\left(x_{1}, x_{2}\right)^{\sigma / v}$, we thus have

$$
\left|u_{2}\left(t, x_{1}\right)-u_{2}\left(t, x_{2}\right)\right| \leq C d\left(x_{1}, x_{2}\right)^{\sigma / v} .
$$

Combining (4.8), (4.11) and (4.12), we conclude that

$$
\left|u\left(t, x_{1}\right)-u\left(t, x_{2}\right)\right| \leq C d\left(x_{1}, x_{2}\right)^{\theta_{1} \sigma /\left(\theta_{1}+\nu \beta\right)} .
$$


for all $t \in(0, T)$ and $x_{1}, x_{2} \in M$ with $d\left(x_{1}, x_{2}\right) \leq 1$, for some $C>0$, where we have used that

$$
\theta_{1} \sigma /\left(\theta_{1}+v \beta\right) \leq \sigma / v \leq \sigma\left(\theta_{2}+\beta\right) /\left(\theta_{2}+v \beta\right) .
$$

The proof is complete.

Finally, one may show that if the heat kernel $k$ satisfies $(k 5)$, if $\|f\|_{\infty}<\infty$ and if $\varphi(x)$ satisfies

$$
\left|K_{t+\delta} \varphi(x)-K_{t} \varphi(x)\right| \leq C \delta \quad(\text { for all } t>0, x \in M),
$$

then the essentially bounded weak solution $u$ of (1.7) is Lipschitz continuous in time $t$ on $(0, T) \times M$, that is,

$$
|u(t+\delta, x)-u(t, x)| \leq C_{1} \delta \quad(t \in(0, T), \delta>0, x \in M) .
$$

We omit the details, which are similar to the special case considered in [9].

We note that, unlike the blow-up and the existence, the regularity of solutions is not related to the Hausdorff dimension $\alpha$ and the walk dimension $\beta$.

\section{REFERENCES}

[1] C. Bandle, H. A. Levine and Qi S. Zhang, Critical exponents of Fujita type for inhomogeneous parabolic equations and systems, J. Math. Anal. Appl. 251, 624648(2000).

[2] M.T. Barlow, Diffusions on Fractals, Lect. Notes Math. 1690, Springer, 1998, 1-121.

[3] M.T. Barlow and R.F. Bass, Brownian motion and harmonic analysis on Sierpínski carpets, Canad. J. Math. (4) 51 (1999), 673-744.

[4] M.T. Barlow, R.F. Bass, Z.-Q. Chen and M. Kassmann, Non-local Dirichlet forms and symmetric jump processes, Trans. Amer. Math. Soc. 361 (2009), 1963-1999.

[5] M.T. Barlow and E.A. Perkins, Brownian motion on the Sierpínski gasket, Probab. Theory. Related Fields 79 (1988), 543-623.

[6] Z.-Q. Chen and T. Kumagai, Heat kernel estimates for jump processes of mixed types on metric measure spaces, Probab. Theory Relat. Fields, 140 (2008), 277-317.

[7] K. Dalrymple, R.S. Strichartz and J.P. Vinson, Fractal differential equations on the Sierpínski gasket, J. Fourier Anal. Appl. 5 (1999), 203-284.

[8] K.J. Falconer, Semilinear PDEs on self-similar fractals, Comm. Math. Phys. 206 (1999), 235-245.

[9] K.J. Falconer and J. Hu, Nonlinear diffusion equations on unbounded fractal domains, J. Math. Anal. Appl. 256 (2001), 606-624.

[10] H. Fujita, On the blowing up of solutions of the Cauchy problem for $u_{t}=\Delta+u^{1+\alpha}$, J. Fac. Sci. Univ. Tokyo Sect. I 13, 109-124(1966).

[11] A. Grigor'yan, J. Hu and Ka-Sing Lau, Heat kernels on metric measure spaces and an application to semilinear elliptic equations, Trans. Amer. Math. Soc. 355, 20652095(2003).

[12] B.M. Hambly and T. Kumagai, Transition density estimates for diffusion processes on post critically finite self-similar fractals, Proc. London Math. Soc. (3)79 (1999), 431-458.

[13] J. Kigami, Analysis on Fractals, Cambridge University Press, 2001.

[14] R.S. Strichartz, Differential Equations on Fractals : a Tutorial, Princeton University Press, 2006.

[15] F.B. Weissler, Local existence and nonexistence for semilinear parabolic equations in $L^{p}$, Indiana Univ. Math. J. 29 (1980), 79-102. 
[16] F.B. Weissler, Existence and non-existence of global solutions for a semilinear heat equation, Israel J. Math. 38 (1981), 29-40.

[17] Q.S. Zhang, A new critical phenomenon for semilinear parabolic problems, J. Math. Anal. Appl. 219(1998), 125-139.

Mathematical Institute, University of St Andrews, North Haugh, St Andrews, Fife KY16 9SS, UK.

E-mail address: kjf@st-andrews.ac.uk

Department of Mathematical Sciences, Tsinghua University, Beijing 100084, China.

E-mail address: hujiaxin@mail.tsinghua.edu.cn

Department of Mathematical Sciences, Tsinghua University, Beijing 100084, China.

E-mail address: sunyh08@mails.tsinghua. edu.cn 